\title{
Location-Aware Spectrum and Power Allocation in Joint Cognitive Communication-Radar Networks
}

\author{
Yogesh Nijsure and Yifan Chen \\ Newcastle University \\ Newcastle upon Tine, UK \\ Email: \{y.nijsure;yifan.chen2\}@ncl.ac.uk
}

\author{
Chau Yuen \\ Singapore University of \\ Technology and Design, Singapore \\ Email: yuenchau@sutd.edu.sg
}

\author{
Yong Huat Chew \\ Institute for Infocomm Research \\ Singapore \\ Email: chewyh@i2r.a-star.edu.sg
}

\begin{abstract}
A novel approach to spectrum and power allocation is proposed for joint cognitive communication-radar networks, which aim at integrating cognitive radio (CR-o) and cognitive radar (CR-r) paradigms to achieve intelligent utilization of spectrum resources in wireless networks. This approach exploits the location information offered by CR-r combined with spectrum sensing capability of CR-o to aid spectrum and power allocation by minimizing harmful interference among neighboring devices. Such systems require both coexistence and sharing of perception of radio environment and radar scene. To offer better spectrum resource utilization, entropy of the received signal is employed in order to detect spectrum holes over the network topology. This entropy-based technique also demonstrates superior performance as compared to the conventional method based on energy detection. Simulation results indicate both throughput improvement and interference reduction among neighboring devices.
\end{abstract}

\section{INTRODUCTION}

Dynamic spectrum access allows frequency bands to be assigned based on the needs of radios without causing significant interference and degradation to the overall throughput. The concept of system cognition proves to be an effective way for intelligent spectrum management in wireless networks [1], [2]. The design and implementation of cognitive radios (CRo) that constantly adapt their operational modes according to the changing radio environment are therefore necessary to utilize the spectrum more efficiently [3]. In terms of CR-o, primary users (PUs) of spectrum resources are referred to as the authorized users of the radio channels and secondary users (SUs) compete for the channels when PUs are inactive [1], [2]. Hence, one of the most important challenges of CR-o is spectrum hole detection, which is to acquire awareness of the frequency usage and existence of PUs in neighboring bands. On the other hand, when SUs are using the vacant channels, CR-o can also be aware of initialization of any primary communication activities in their vicinity. The detection of PUs is very important, not only to prevent interference but also to detect any opportunity of communication for SUs as shown in [1].

Location information of CR-o can prove to be beneficial in identifying spectrum holes over the network, which can be used to assist in spectrum allocation in order to avoid interference among users in close vicinity. This information can be obtained from a dedicated cognitive radar (CR-r) network as discussed in [4], [5]. This motivates the design of a novel cognitive radio radar (CRR) system presented in the current work. Essentially, we combine the functionalities of CR-o and CR-r to facilitate localization as well as intelligent spectrum and power allocation. Specifically, in a CRR network, radar targets are also radio users, which results in coexistence of radio environment and radar scene. Furthermore, the knowledge about the location and identification of a specific radar target, which uses certain radio channels for communications, can be fed into the CR-o network to assist in decision making about spectrum assignment strategies. Similarly, the CR-o network can also localize and identify the radio users by analyzing the received signal. This information can be fed into the CR$r$ network to assist in radar waveform design and selection, target state estimation, and power allocation [4]-[7]. This leads to sharing of perception of radio and radar scenes under surveillance.

The key contributions of this paper are:

1) Development of an algorithm based upon entropy of the received signal for spectrum sensing;

2) Integration of CR-o and CR-r concepts to form a CRR network, which achieves location-aware spectrum and power management; and

3) Evaluation of the system throughput and comparison of the proposed method with the conventional energy-based detection in terms of true positive rates.

The rest of the paper is organized as follows. In Section II, we describe the proposed CRR network architecture and the entropy-based spectrum sensing. In Section III, we describe the algorithm for spectrum and power allocation among the CR-o users. In Section IV, we present the simulation results on the system throughput and also comparison with the conventional detection technique based on the received signal energy. Finally, some concluding remarks are drawn in Section $\mathrm{V}$.

\section{CRR NeTwORK ARCHItecture AND ENTROPY-BASED SPECTRUM SENSING}

The spectrum mask defined by IEEE 802.11 b requires that the signal is attenuated by at least $30 \mathrm{~dB}$ from its peak energy at $\pm 11 \mathrm{MHz}$ from the center frequency, and attenuated by at least $50 \mathrm{~dB}$ from its peak energy at $\pm 22 \mathrm{MHz}$ from the center frequency [2]. Since the spectrum mask only defines power output restrictions up to $\pm 22 \mathrm{MHz}$ from the center frequency, 




Fig. 1. CRR network architecture.

it is often assumed that the energy of the channel extends no further than these limits. IEEE $802.11 \mathrm{~b}$ also divides the spectrum into 14 overlapping, staggered channels whose center frequencies are $5 \mathrm{MHz}$ apart. Given the separation between channels 1,6 , and 11 , signals sent by a radio occupying any channel could be sufficiently attenuated to minimally interfere with other neighboring radios utilizing the other two frequency bands. Hence, we will consider these 3 channels for spectrum allocation in this work. If transmitters are closer together than channels 1,6 , and 11 (e.g., 1, 4, 7, and 10), overlap between the channels will probably cause unacceptable degradation of signal quality and throughput.

The proposed CRR network is illustrated in Fig. 1. The system consists of cognitive base stations (CBSs) at fixed locations and cognitive mobile stations (CMSs) sharing 3 nonoverlapping channels 1,6 and 11 under IEEE $802.11 \mathrm{~b}$. A CBS comprises a joint communication and radar platform (see e.g., the system concepts proposed in [8], [9]). On the other hand, a CMS is a radio user as well as a radar target. For example, a radio frequency identification (RFID) tag could be attached to a CMS for enhanced and recognizable radar cross section [4]. Subsequently, the radar components in CBSs transmit probing waveforms, which are reflected by CMSs. The relevant information for positioning such as received signal strength, time-of-arrival, and angle-of-arrival is recorded at CBSs and forwarded to a cognitive engine (CE) through wired links, which is the centralize decision-making entity as shown in Fig. 1. The CE then works out the locations of CMSs by triangulation [10]. It is worth emphasizing that, cognitive operations based on the experience gained from the recognized radar-returns from CMSs could be implemented following the CR-r concepts developed in [4], [5], which include: (i) adaptive and continuous allocation of limited transmit power among the activated CBSs; (ii) intelligent illumination of the environment through antenna beamsteering at CBSs; and (iii) adjusting the set of activated CBSs to achieve the optimized
CMS detection and localization.

Subsequently, the spectrum holes are identified by using an information-theoretic measure known as entropy of the received signal. Conventional spectrum sensing methods include energy detection [11], matched filtering [12], and cyclostationarity-based detection. Owing to its low computational complexity, energy detection algorithm is commonly used. This method requires knowledge of noise and signal powers. Nevertheless, the noise may not be stationary and its variance may not be known a priori. On the other hand, matched filtering is the optimum method for spectrum hole detection. However, it demands full knowledge of the signaling features such as bandwidth, operating frequency, modulation type and order, pulse shaping, etc. As a result, this method is not suitable for practical usage.

The entropy-based spectrum sensing can distinguish signals from noises because it is known that a stochastic signal with Gaussian probability density function (pdf) has the maximum entropy [13]. This detection method has been proposed recently [14] and has been proved to be intrinsically robust against noise uncertainty. The existence of radio users over a channel is established when the entropy of the received signal is smaller than a prescribed threshold. Each CMS constantly performs spectrum sensing over the 3 available channels and notifies the availability of a particular channel to the CE through the radio links with the CBSs as depicted in Fig. 1. The CE then decides upon the optimal spectrum allocation strategy and determines the corresponding operational parameters like transmission power for each CMS by utilizing the location information in order to minimize interference and enhance overall system throughput. Eventually, this enables an interference-suppressed secondary radio link between each pair of CMS and its neighboring SU as shown in Fig. 1. For simplicity, we will assume that the SU is not a CMS (i.e., it is a radio user but not a radar target).

We now present the analysis of the entropy-based spectrum sensing undertaken by CMSs. This method establishes the presence or absence of a user over a radio channel based upon hypothesis testing as follows

$$
\begin{array}{ll}
H_{0}: s(n)=u(n), & n=0,1, \ldots, K-1 \\
H_{1}: s(n)=y(n)+u(n), & n=0,1, \ldots, K-1
\end{array}
$$

where $H_{0}$ and $H_{1}$ denote the absence and presence of the user, respectively. $s(n)$ is the received signal, $y(n)$ indicates the transmitted signal from the user, $u(n)$ is the additive white Gaussian noise (AWGN), and $K$ denotes the number of samples over the observation period. Therefore, the distribution of $s(n)$ follows the Gaussian pdf as

$$
\begin{aligned}
& H_{0}: s(n) \sim \mathcal{N}\left(0, \sigma_{u}^{2}\right) \\
& H_{1}: s(n) \sim \mathcal{N}\left(\mu, \sigma_{y}^{2}+\sigma_{u}^{2}\right)
\end{aligned}
$$

where $\mathcal{N}(\cdot, \cdot)$ denotes the normal distribution, $\sigma_{u}^{2}$ is the noise variance, and $\mu$ and $\sigma_{y}^{2}$ are the mean and variance of the signal, respectively. 
Applying discrete Fourier transform to (1) and (2), we obtain

$$
\begin{array}{ll}
H_{0}: \mathbf{S}(k)=\mathbf{U}(k), & k=0,1, \ldots, K-1 \\
H_{1}: \mathbf{S}(k)=\mathbf{Y}(k)+\mathbf{U}(k), & k=0,1, \ldots, K-1
\end{array}
$$

where $\mathbf{S}(k), \mathbf{Y}(k)$, and $\mathbf{U}(k)$ represent the complex vectors of received signal, transmitted signal, and noise, respectively. Alternatively, $\mathbf{S}(k)$ can also be written as

$$
\mathbf{S}(k)=\frac{1}{K} \sum_{n=0}^{K-1} s(n) \exp \left(-j \frac{2 \pi}{K} k n\right)
$$

The detection strategy involves testing of the information entropy $\mathcal{H}(\mathbf{S})$, which is a measure of uncertainty associated with the random signal $\mathbf{S}$, defined as

$$
\mathcal{H}(\mathbf{S})=-\sum_{i=1}^{I} p\left(S_{i}\right) \log _{2} p\left(S_{i}\right)
$$

In the preceding equation, $I$ is the total number of countable states, often referred to as the dimension of the probability space. $p\left(S_{i}\right)$ denotes the probability of observation of a received signal level, $S_{i}$, such that $\sum_{i=1}^{I} p\left(S_{i}\right)=1$. We use the histogram method for entropy estimation as suggested in [14]. Let $o_{i}$ be the number of occurrences of received signal strength falling in the $i$ th bin, Hence, $p\left(S_{i}\right) \approx o_{i} / O$ with $O$ being the total number of occurrences. The bin width $\delta$ can be defined as

$$
\delta=\frac{S_{\max }-S_{\min }}{I}
$$

where $S_{\max }$ and $S_{\min }$ are the maximum and minimum values of the received signal strength. Following (3) and (5), the estimated entropy (or the test statistic) is given by

$$
\lambda(\mathbf{S})=\mathcal{H}(\mathbf{S})=-\sum_{i=1}^{I} \frac{o_{i}}{K} \log _{2} \frac{o_{i}}{K}
$$

Subsequently, we utilize the above test statistic to evaluate the two hypotheses for spectrum user detection

$$
\begin{aligned}
& H_{0}: \lambda(\mathbf{S})>\lambda_{0} \\
& H_{1}: \lambda(\mathbf{S}) \leq \lambda_{0}
\end{aligned}
$$

where $\lambda_{0}$ is the threshold determined by the probability of false alarm. The value of $\lambda$ will decrease according to the convex property of information entropy as the channel changes its state from "free" to "busy". In the next section we will describe the algorithm for adapting transmission parameters of CMSs once spectral holes are identified.

\section{Spectrum And Power Allocation Algorithm}

As described earlier, all the CMSs perform entropy-based spectrum sensing on the 3 available radio channels and notify the $\mathrm{CE}$ about spectrum holes detected in their neighboring regions. The CE takes into consideration the physical locations of all the CMSs acquired through the radar units of the $\mathrm{CBSs}$, and allots the available frequencies with an intention of minimizing interference among neighboring CMSs. It instructs each CMS to occupy a vacant channel and decides upon the transmission power for each CMS with an objective of enhancing the overall system throughput. The capacity of the radio link between two radio users can be expressed as [15], [16]

$$
C(\rho, \eta)=B \ln \left(1+\frac{\rho}{1+\eta}\right)
$$

where $C(\rho, \eta)$ denotes the capacity of the radio link as a function of the signal-to-noise ratio (SNR) $\rho$, the interference-tonoise ratio (INR) $\eta$, and the channel bandwidth $B$. Therefore, the total capacity (or throughput) of the CRR network can be calculated as

$$
\begin{aligned}
\bar{C} & =\sum_{l=1}^{3} \sum_{m=1}^{M} C\left(\frac{\alpha_{m m}(l) P_{m}(l)}{\sigma_{u}^{2}}, \frac{\sum_{n \neq m} \alpha_{m n}(l) P_{n}(l)}{\sigma_{u}^{2}}\right) \\
& =\sum_{l=1}^{3} \sum_{m=1}^{M} C\left(\rho_{m}(l), \eta_{m}(l)\right), \quad n \in\{1,2, \cdots, M\}
\end{aligned}
$$

In the preceding equation, $l \in\{1,2,3\}$ is the index of the available channel, $M$ is the total number of point-topoint communication links using a particular channel, $\alpha_{m m}(l)$ represents the channel gain of link $m$ using channel $l, \alpha_{m n}(l)$ represents the channel gain between the transmitter of link $n$ and the receiver of link $m, P_{m}(l)$ and $P_{n}(l)$ are the transmission powers for link $m$ and link $n$, respectively. $\rho_{m}(l)=$ $\alpha_{m m}(l) P_{m}(l) / \sigma_{u}^{2}$ and $\eta_{m}(l)=\sum_{n \neq m} \alpha_{m n}(l) P_{n}(l) / \sigma_{u}^{2}$ are the SNR and INR of link $m$ occupying channel $l$, respectively. Note that the same wireless link may utilize multiple channels for data communications.

Next, the transmission power of each link is subject to the following constraint

$$
\sum_{l=1}^{3} P_{m}(l) \leq P_{0}, \quad \forall m
$$

We adopt the Lagrange multiplier method in order to derive the power allocation strategy. The stationary point on $\bar{C}$ is found using

$$
\frac{\partial \bar{C}}{\partial P_{m}(l)}=\sum_{l=1}^{3} \sum_{m=1}^{M} \frac{\partial C\left(\rho_{m}(l), \eta_{m}(l)\right)}{\partial P_{m}(l)}=0
$$

Furthermore, following (11) and (12), we have $\partial C / \partial P_{m}(l)=$ $\gamma$ (a constant for a particular link). Subsequently, applying the capacity function in (10) yields

$$
\begin{aligned}
& \frac{\partial C\left(\rho_{m}(l), \eta_{m}(l)\right)}{\partial P_{m}(l)} \\
&=\frac{\partial}{\partial P_{m}(l)}\left\{B \ln \left[1+\frac{\rho_{m}(l)}{1+\eta_{m}(l)}\right]\right\} \\
&=\frac{B}{1+\frac{\rho_{m}(l)}{1+\eta_{m}(l)}} \times\left[\frac{1}{1+\eta_{m}(l)} \frac{\partial \rho_{m}(l)}{\partial P_{m}(l)}\right. \\
&\left.-\frac{\rho_{m}(l)}{\left[1+\eta_{m}(l)\right]^{2}} \frac{\partial \eta_{m}(l)}{\partial P_{m}(l)}\right]
\end{aligned}
$$


As the INR $\eta_{m}(l)$ is not a function of the transmission power $P_{m}(l)$, the above equation can be further simplified by substituting the expressions of $\rho_{m}(l)$ and $\eta_{m}(l)$ :

$$
\frac{B \alpha_{m m}(l)}{\sigma_{u}^{2}+\sum_{n \neq m} \alpha_{m n}(l) P_{n}(l)+\alpha_{m m}(l) P_{m}(l)}=\gamma
$$

Rearranging the above equation yields

$$
P_{m}(l)=\frac{B}{\gamma}-\frac{\sigma_{u}^{2}+\sum_{n \neq m} \alpha_{m n}(l) P_{n}(l)}{\alpha_{m m}(l)}
$$

This is similar to the water-filling approach. We then solve (15) iteratively in order to find the profile of $P_{m}(l)$ across all the available channels $(l=1,2,3)$ for link $m$.

The algorithm can be summarized as follows (see also Fig. 1).

1) All the CMSs in the CRR network maintain active radar links with the CBSs.

2) The CBSs extract the propagation parameters relevant to positioning by analyzing the backscatter signals from the CMSs, and forward the information to the CE in order to perform centralized CMS localization.

3) The CMSs also inform the CE about the availability of vacant channels using (8) in their neighboring regions. This message is sent through the radio links between the CMSs and CBSs.

4) The CE calculates the optimum spectrum and transmission power allocation strategies for each CMS, which are forwarded to all the CMSs via the radio links between the CBSs and CMSs.

5) Each CMS then establishes an interference-suppressed radio link with the SU within its exclusive coverage area.

6) As the CMSs are mobile, the preceding steps are repeated to dynamically update the transmission parameters of each CMS.

\section{SIMULATION RESULTS}

For the purpose of simulation, we assume a square grid of $10 \times 10$ CMSs sharing the 3 available channels under IEEE 802.11 b. Fig. 2 indicates the initial random channel assignment over the CMSs. Each CMS occupies a square footprint and communicates with an SU within this exclusive coverage area. Each color (blue, red, or yellow) represents one of the three available channels (1,6 and 11). The transmission power for each CMS is uniformly distributed between 0 and $P_{0}$ for the first-stage power allocation. The area of dark circle at the center of each square footprint represents the transmission power of the corresponding CMS. Apparently, the initial channel assignment is susceptible to severe interference as can be seen from Fig. 2, where a large number of neighboring cells utilize the same radio channel for communications.

Next, each CMS constantly maintains radar links with the CBSs. The propagation parameters such as received signal strength, time-of-arrival, and angle-of-arrival obtained at more than 3 non-collocated CBSs can be used to localize the CMSs. For simplicity, it is assumed that the CMS locations have been successfully estimated (e.g., by following the methodologies

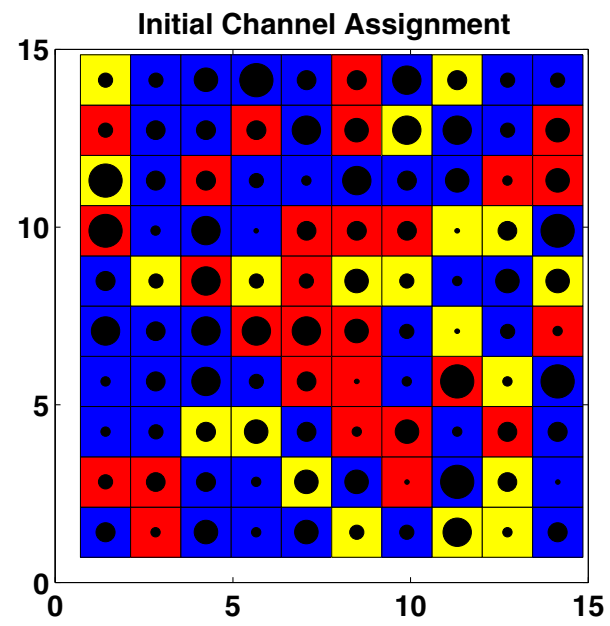

Fig. 2. Initial random channel assignment.

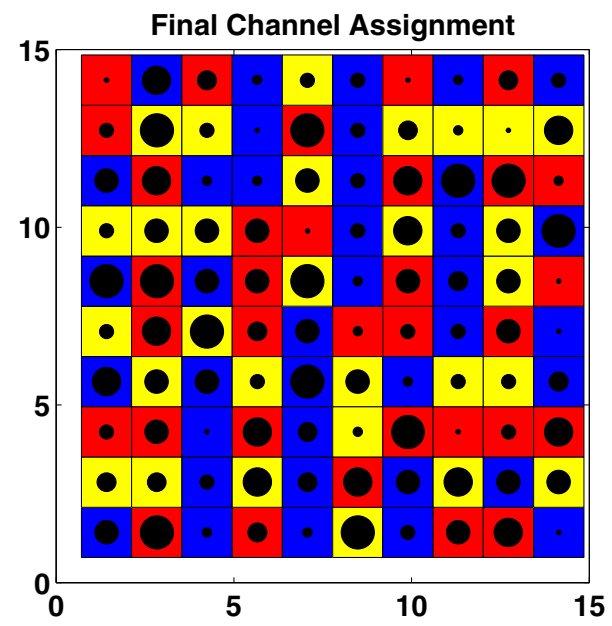

Fig. 3. Final location-aware channel assignment.

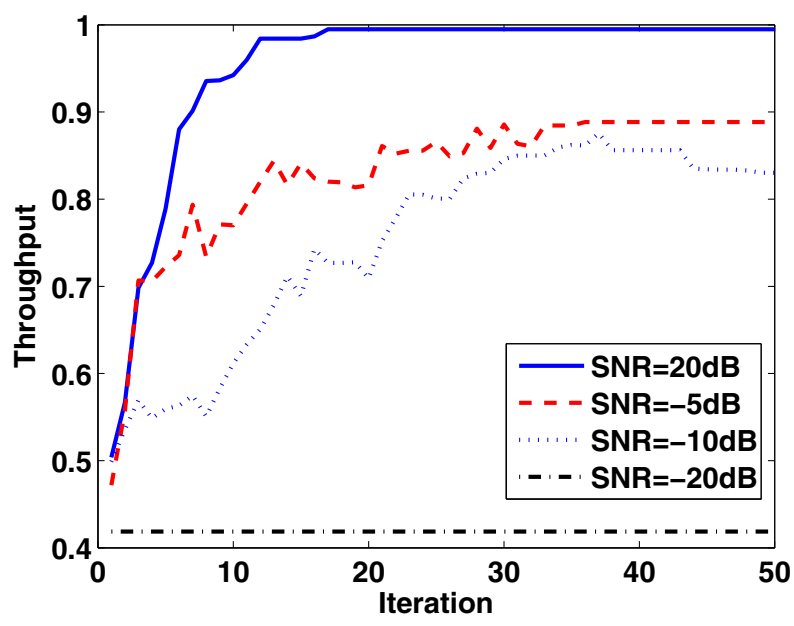

Fig. 4. CRR network throughput. 


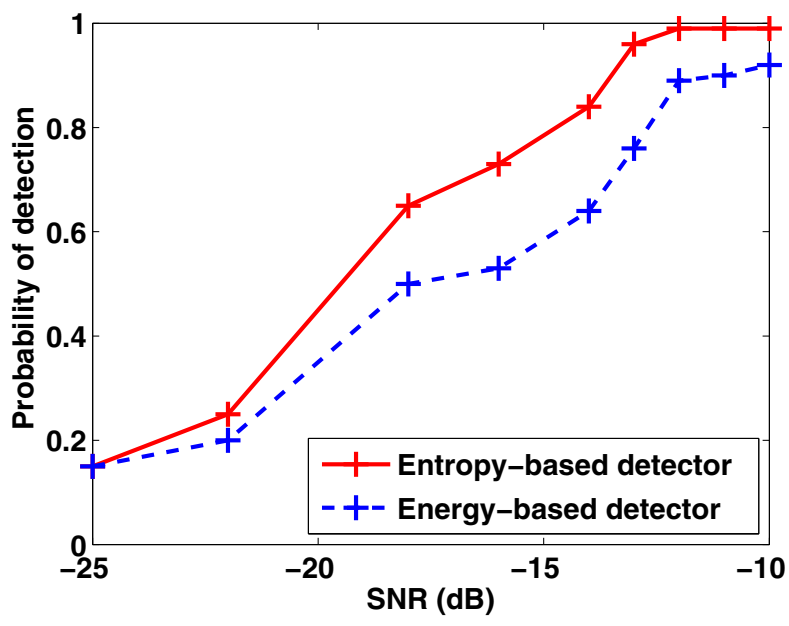

Fig. 5. Performance of spectrum user detection.

proposed in [4], [5]). Once the location of each CMS is established, the CE decides upon the spectrum allocation strategy based on the knowledge of spectrum holes or vacant channels around each CMS, which is imparted to the CE via the radio links between CMS and CBS.

Subsequently, the transmission power of each CMS is adjusted iteratively as per the power allocation algorithm described in Section III. We consider a simplified interference scenario, where the two channel gains $\alpha_{m m}$ and $\alpha_{m n}$ in (10) are assumed to be two fixed constants. Furthermore, only the first-tier interferers (i.e., for each CMS under consideration, its immediate 8 neighboring cells) are taken into account for power allocation using (15). The main intention of this spectrum and power allocation algorithm is to avoid clustering of CMSs using the same channel, and to distribute transmission power according to the water-filling approach in order to enhance the overall throughput. Fig. 3 indicates the final channel assignment. In this case, we observe lower number of neighboring CMSs sharing the same channel. This indicates reduction of interference among CMSs.

Fig. 4 illustrates the throughput of the overall system. Each characteristics curve in this plot represents a throughput at a particular value of SNR. It can be seen that the throughput converges after a certain number of iterations for each SNR. In general, faster convergence is achieved for higher SNRs. Nevertheless, in the case of very low SNR (SNR $=-20 \mathrm{~dB})$, more iterations fail to result in improved throughput.

Finally, Fig. 5 demonstrates the performance of the entropybased detector as compared against the energy-based detector. These results have been generated by averaging over 100 simulations at different SNRs. The probability of false alarm has been fixed at 0.15 for evaluating the value of the threshold $\lambda_{0}$ in (8). As discussed previously, the entropy-based detector does not require any previous knowledge about transmission parameters of the signal or noise characteristics. It is also quite robust and insensitive to noise uncertainty. Thus its performance is superior as compared to the energy-based detection in terms of probability of spectrum user detection even at lower SNRs as can be seen from Fig. 5.

\section{CONClusion}

We have presented the integration of CR-o and CR-r concepts in order to facilitate efficient spectrum and power allocation among CMSs in the CRR network. Simulation results have demonstrated superior performance of the proposed algorithm in terms of interference reduction and throughput enhancement. Location-aware power and spectrum allocation by using a joint cognitive communication-radar network seems to offer increased adaptability as compared to the traditional decoupled radio and radar systems. Also the integration of these two concepts opens up new possibilities for implementation of improved sensing, detection, cooperative communications, and intelligent signal processing for various wireless applications.

\section{ACKNOWLEDGEMENT}

This research is partly supported by the International Design Center (Grant No. G3.2 and D1.3).

\section{REFERENCES}

[1] S. Haykin, "Cognitive radio: brain-empowered wireless communications," IEEE J. Sel. Areas Commun., vol. 23, no. 2, pp. 201-220, 2005.

[2] Federal Communications Commission Spectrum Efficiency Working Group, "Spectrum Policy Task Force Report," FCC Report, Nov. 2002.

[3] J. Mitola and G. Q. Maguire, "Cognitive radio: making software radios more personal," IEEE Personal Communications, vol. 6, no. 4, pp. 13 18, 1999.

[4] Y. Chen and P. Rapajic, "Ultra-wideband cognitive interrogator network: Adaptive illumination with active sensors for target localisation," IET Commun., vol. 4, no. 5, pp. 573-584, 2010.

[5] Y. Nijsure, Y. Chen, C. Litchfield, and P. Rapajic, "Hidden Markov model for target tracking with UWB radar systems," in Proc. IEEE 20th Personal, Indoor and Mobile Radio Communications (PIMRC 2009), Sep. 2009, pp. 2065-2069.

[6] S. Haykin, "Next generation of radar systems enabled with cognition," in Proc. IEEE Radar Conference, May 2010, pp. 1-1.

[7] H. Celebi, I. Guvenc, S. Gezici, and H. Arslan, "Cognitive-radio systems for spectrum, location, and environmental awareness," IEEE Antennas Propag. Mag., vol. 52, no. 4, pp. 41-61, 2010.

[8] D. Garmatyuk, J. Schuerger, Y. Morton, K. Binns, M. Durbin, and J. Kimani, "Feasibility study of a multi-carrier dual-use imaging radar and communication system," in Proc. European Radar Conference (EURAD 2007), Oct. 2007, pp. 194-197.

[9] C. Sturm, T. Zwick, and W. Wiesbeck, "An OFDM system concept for joint radar and communications operations," in Proc. IEEE 69th Veh. Technol. Conf. (VTC Spring 2009), Apr. 2009, pp. 1-5.

[10] M. L. Skolnik, Introduction to Radar Systems, 3rd Ed., McGraw-Hill, New York, 2001.

[11] H. Urkowitz, "Energy detection of unknown deterministic signals," Proceedings of the IEEE, vol. 55, no. 4, pp. 523-531, 1967.

[12] F. F. Digham, M. S. Alouini, and M. K. Simon, "On the energy detection of unknown signals over fading channels," IEEE Trans. Commun., vol. 55, no. 1, pp. 21-24, 2007.

[13] X. Chen and S. Nagaraj, "Entropy based spectrum sensing in cognitive radio," in Proc. Wireless Telecommunications Symposium (WTS 2008), Apr. 2008, pp. 57-61

[14] Y. L. Zhang, Q. Y. Zhang, and T. Melodi, "A frequency-domain entropybased detector for robust spectrum sensing in cognitive radio networks,' IEEE Commun. Lett., vol. 14, no. 6, pp. 533-535, June 2010.

[15] C. E. Shannon, "Communication in the presence of noise," Proceedings of the IRE, vol. 37, no. 1, pp. 10-21, 1949.

[16] A. G. Burr, "Capacity of cognitive channel and power allocation," in Proc. Information Theory Workshop (ITW 2009), Oct. 2009, pp. 510 514. 